\title{
High-fertility phenotypes: two outbred mouse models exhibit substantially different molecular and physiological strategies warranting improved fertility
}

\author{
Martina Langhammer ${ }^{2, *}$, Marten Michaelis ${ }^{1, *}$, Andreas Hoeflich $^{3}$, Alexander Sobczak $^{1}$, \\ Jennifer Schoen ${ }^{4}$ and Joachim M Weitzel ${ }^{1}$ \\ ${ }^{1}$ Institut für Fortpflanzungsbiologie, ${ }^{2}$ Institut für Genetik und Biometrie and ${ }^{3}$ Institut für Genombiologie, \\ Leibniz-Institut für Nutztierbiologie (FBN), Wilhelm-Stahl-Allee 2, 18196 Dummerstorf, Germany and \\ ${ }^{4}$ Berlin-Brandenburg School for Regenerative Therapies, Charité, Berlin, Germany \\ Correspondence should be addressed to J M Weitzel; Email: weitzel@fbn-dummerstorf.de \\ ${ }^{*}$ (M Langhammer and M Michaelis contributed equally to this work)
}

\begin{abstract}
Animal models are valuable tools in fertility research. Worldwide, there are more than $\mathbf{4 0 0}$ transgenic or knockout mouse models available showing a reproductive phenotype; almost all of them exhibit an infertile or at least subfertile phenotype. By contrast, animal models revealing an improved fertility phenotype are barely described. This article summarizes data on two outbred mouse models exhibiting a 'high-fertility' phenotype. These mouse lines were generated via selection over a time period of more than 40 years and 161 generations. During this selection period, the number of offspring per litter and the total birth weight of the entire litter nearly doubled. Concomitantly with the increased fertility phenotype, several endocrine parameters (e.g. serum testosterone concentrations in male animals), physiological parameters (e.g. body weight, accelerated puberty, and life expectancy), and behavioral parameters (e.g. behavior in an open field and endurance fitness on a treadmill) were altered. We demonstrate that the two independently bred high-fertility mouse lines warranted their improved fertility phenotype using different molecular and physiological strategies. The fertility lines display female- as well as male-specific characteristics. These genetically heterogeneous mouse models provide new insights into molecular and cellular mechanisms that enhance fertility. In view of decreasing fertility in men, these models will therefore be a precious information source for human reproductive medicine.
\end{abstract}

\section{Translated abstract}

A German translation of abstract is freely available at http://www.reproduction-online.org/content/147/4/427/suppl/DC1.

Reproduction (2014) 147 427-433

\footnotetext{
This paper forms part of a special issue of Reproduction on Endocrine Disrupters. This article was presented at the 7th Copenhagen Workshop on Endocrine Disrupters, 28-31 May 2013. The meeting was supported by the Danish Ministry of the Environment - Environmental Protection Agency as an activity under the Danish Centre on Endocrine Disrupters. Publication of this special issue has been supported by the Society for Reproduction and Fertility. The opinions or views expressed in this special issue are those of the authors, and do not necessarily reflect the opinions or recommendations of the Danish Ministry of the Environment - Environmental Protection Agency or the Society for Reproduction and Fertility. The Guest Editors for this special issue were Anna-Maria Andersson, Hanne Frederiksen, Niels Erik Skakkebæk, Rigshospitalet, Denmark, Kenneth M Grigor, Western General Hospital, Edinburgh, UK and Jorma Toppari, University of Turku, Finland.
}

\section{Introduction}

\section{Animal models for fertility research}

Fertility research is largely supported by the use of informative animal models. Most of these animal models are mouse lines. Worldwide, there are more than 400 transgenic or knockout mouse lines available showing a reproductive phenotype (Matzuk \& Lamb 2008, Jamsai \& O'Bryan 2011, Ogorevc et al. 2011). Almost all of them exhibit an infertile or at least subfertile phenotype. The infertile or rather subfertile characteristics of a yet unelucidated novel mouse model are readily detectable in the context of standard animal care conditions due to obvious difficulties in breeding these animals. In general, mouse models revealing reduced fertility are unquestionably helpful in identifying crucial proteins that 
interfere with any critical step necessary for proper fertility in man and/or animal both in males and in females. Furthermore, these mouse lines are undoubtedly helpful in detecting and dissecting fertility-relevant pathways.

However, infertility in man appears to be more complex and as such does not affect only one single gene but rather a combination of multiple genes. Additionally, it might be an oversimplification to divide all fertility phenotypes into only two categories, i.e. fertile and infertile. A more subtle differentiation might be more appropriate to reflect the diverse fertility phenomena. Furthermore, it might be valuable to involve and pursue more comprehensive parameters for analyzing fertility (e.g. partner, environment, and endocrine disruptors). To this end, animal models showing an improved fertility phenotype with an inherent illustration of the complex and biodiverse fertility nature might become highly informative.

\section{Animal models with a high-fertility phenotype}

In contrast to animal models revealing diminished fertility, the reciprocal phenotype (high fertility) is not easily identified during standard animal care conditions. To open up this bottleneck, breeding protocols have been set up to select for high-fertility traits. Such protocols have been conducted in several animal species so far, e.g. mouse, pig, and rabbit (Johnson et al. 1999, Holt et al. 2004, Su et al. 2007, Ziadi et al. 2013).

Particularly, establishing a high-fertility model for pigs is considerably relevant for farm animal biology. This is pointed out by the circumstance that breeding over the last several 1000 years has led to a rise in the average litter size in pigs from two to eight piglets in wild boars to more than 15 piglets in contemporary domestic pigs (Su et al. 2007, Beaulieu et al. 2010). Although this litter size is economically desirable, its generation by breeding is currently associated with major problems resulting from highly unbalanced birth weights of piglets ranging from $<1 \mathrm{~kg}$ (very small piglets) to $>1.8 \mathrm{~kg}$ (very large piglets). Hence, this heterogeneous piglet population leads to higher and unintended follow-up breeding costs (Rehfeldt et al. 2008, Beaulieu et al. 2010). This can be avoided by achieving homogeneous litters with a size of more than 15 piglets and a balanced birth weight of $\sim 1.3-1.5 \mathrm{~kg} /$ piglet. As our high-fertility mouse models generate homogeneous litters despite high offspring numbers, they might be of great value to elucidate physiological mechanisms and target genes responsible for this desired property.

\section{Mouse models for high-fertility traits}

To examine physiological and genetic responses to selection for fertility traits, long-term selection mouse lines were established as models for farm animals at the Leibniz Institute for Farm Animal Biology (Dummerstorf), which were selected for the phenotype 'high fertility'. All the animal experiments were approved by local authorities (Landkreis Bad Doberan, Veterinär- und Lebensmittelüberwachungsamt, Mecklenburg-Vorpommern, Germany). The initial population was a systematic crossbreed of four inbred and four outbred founder mouse lines starting in the 1970s (Dietl et al. 2004). As selection criterion, a fecundity index was set up comprising both i) number of offspring per litter and ii) total birth weight of the entire litter (Dummerstorf fecundity index $=1.6 \times$ number of offspring + birth weight of the entire litter). Animals born from the largest and heaviest litters were chosen for further breeding (Schüler \& Bünger 1982). Whereas fertility line 1 (FL1) was treated, the estrus of the females in FL2 was synchronized by application of the gestagen chlormadinone acetate up to the twenty-third generation. Thus, two independent mouse lines were developed. The resulting FLs (FL1 and FL2) were maintained with a population size of 60-100 animals per generation; consequently, these outbred mouse lines are more heterogeneous and biodiverse in nature compared with classical inbred mouse lines. Females were exposed to males at an age of 63 days with a mating ratio of 1:1. After more than 40 years and 161 generations of breeding, the litter size increased from approximately ten animals per litter in the original founder population to $17.6 \pm 3.3$ and $20.2 \pm 2.0$ animals per litter in FL1 and FL2 respectively. The litter size of the unselected and randomly mated control line (Ctrl) animals remained largely constant over the selection period. The breeding success during the whole selection proceeding of more than 40 years with regard to the number of born pups per litter is summarized in Fig. 1.

The Dummerstorf mouse lines FL1 and FL2 represent to our knowledge - a worldwide unique animal recourse. Admittedly, a similar long-term breeding approach in mice has been followed by Odd Vangen et al. from the Agricultural University of Norway in Ås

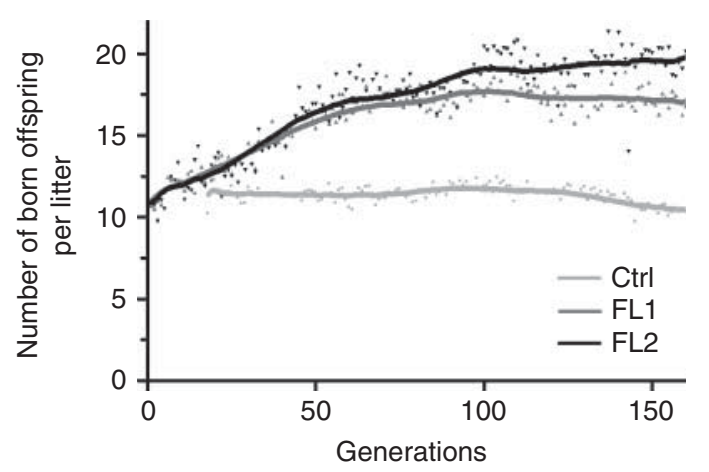

Figure 1 Selection success concerning the mean number of born offspring per litter in FL1 and FL2 during 161 generations of fertility index selection compared with the unselected control line (Ctrl). Data points represent the mean of at least 50 individual litters per generation and mouse line. 
with high-fertility mice selected for more than 110 generations (Holt et al. 2004, 2005). However, this line was terminated in 2007 after 130 generations of selection (Odd Vangen 2013, personal communication). Moreover, reports can be found about experimental attempts to increase fecundity in mice on the basis of inbred lines. One of these inbred models is the QSi5 mouse line created by Peter Williamson et al. from Sydney, Australia (Wei et al. 2013). These mice are distinguished by an average litter size of 13.4 animals.

\section{Breeding success and fecundity indices}

Our Dummerstorf mouse lines were selected according to a fecundity index corresponding to $1.6 \times$ litter size + litter weight on the day of birth. This fertility index was selected to avoid a selection for only high litter sizes with perhaps simultaneously reduced individual (and possibly unhealthy) body weight of the offspring. Thus, this fertility index reflects both parameters: size and birth weight of the entire litter. Other commonly used mouse fecundity indices integrate the following fertility-relevant parameters: i) litter size; ii) litters born per dam (lifetime fecundity); and iii) successful mating rate (Silver 1995). As lifetime fecundity in our mouse lines has - unfortunately not been determined so far, it is somewhat difficult to draw comparisons with these lines on the basis of the Silver fecundity index. However, restricted to the parameters of litter size and successful mating rate, comparisons of FL1/FL2 opposed to QSi5 and other inbred mouse lines revealed similar or actually higher values (Table 1).

It is quite noteworthy that unselected outbred mouse lines (as those employed in our model as the Ctrl) exhibit a significantly higher fecundity with an average litter size of around ten animals (Bowman \& Falconer 1960, Bradford 1979) compared with today's inbred mouse lines (see Table 1). Inbred mouse lines have to be generally considered as subfertile if compared with unselected outbred mouse lines. Thus, it is important to take into account that the majority of investigations of genetically modified mouse models that have been back-crossed to C57BL/6J deal with subfertile control animals. Even those inbred lines that have been thought of as being relatively highly fertile are indeed subfertile in comparison with outbred mice; in particular, even C57BL/6J mice are subfertile (see Table 1). Moreover, it is not clear which reproductive and physiological parameters are altered by inbreeding. This means that inbred mouse lines already exhibit decreased reproductive physiology and might not constitute the best model for investigations regarding proper fertility.

\section{Bias during breeding protocol}

Every selection protocol is prone to select additionally for undesired or initially overlooked side effects. An unintended side effect was observed during the last 115 generations of breeding. The body weight for both genders of the FLs was increased at the time of mating - an effect especially pronounced in FL2 females (Fig. 2A). Additionally, it is worth noting that the common sex dimorphism in body weight inverts in FL2 animals in favor of the females. FL1 females at least reach the same body masses as the male animals. As the selection aimed toward large litters, it can be suggested that an overall growth in body weight of the dams might be helpful for proper delivery. A rise in dam body weight during the selection period has also been noticed in the Norwegian high-FL (Holt et al. 2004).

Furthermore, a shift in growth development and accelerated puberty of FL1 and FL2 animals has been detected. Whereas the time point of vaginal opening did not differ between the Ctrl, FL1, and FL2 animals, we observed an earlier entry into the first estrus for the FLs in contrast to the Ctrl (Fig. 2B). Thus, it could be assumed that the developmental program is accelerated in high-FLs.

\section{Characterization of the FLs FL1 and FL2 on the female side}

The two Dummerstorf FLs FL1 and FL2 were initially analyzed in more detail on the female side. In this context, it became clear that different physiological parameters are altered in both mouse lines. While FL2

Table 1 Fecundity index according to Silver (1995). Summary of litter size, litters born per dam, and successful mating rates in different outbred and inbred mouse lines (for details see text).

\begin{tabular}{|c|c|c|c|c|c|}
\hline Strains & $\begin{array}{c}\text { Litter } \\
\text { size }(n)\end{array}$ & $\begin{array}{c}\text { Litters born } \\
\text { per dam }\end{array}$ & $\begin{array}{l}\text { Successful } \\
\text { mating rate }\end{array}$ & $\begin{array}{c}\text { Fecundity } \\
\text { index }\end{array}$ & References \\
\hline FL1 & 17.6 & ND & 0.92 & ND & This study \\
\hline Ctrl (Dummerstorf) & 10.8 & ND & 0.84 & ND & This study \\
\hline WT & 8.3 & ND & 0.98 & ND & Bradford (1979) \\
\hline Norwegian line & 21.6 & ND & 0.80 & ND & Holt et al. $(2004,2005)$ \\
\hline DBA/2J & 5.4 & 3.9 & 0.75 & 15.8 & Silver (1995) \\
\hline $\mathrm{A} / \mathrm{J}$ & 6.3 & 2.9 & 0.65 & 11.9 & Silver (1995) \\
\hline $\mathrm{BALB} / \mathrm{CJ}$ & 5.2 & 3.8 & 0.47 & 9.3 & Silver (1995) \\
\hline
\end{tabular}

ND, not determined. 

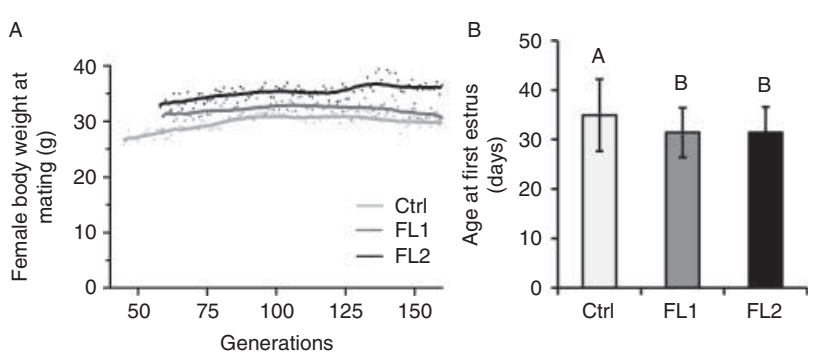

Figure 2 (A) Female body weight at the time of mating in the fertility lines (FLs) FL1 and FL2 compared with the unselected Ctrl. Female animals were measured at 9 weeks of life over the selection period. (B) Time point of first estrus in the FLs FL1 and FL2 as well as in unselected control animals (Ctrl). Data points represent means \pm s.D. of at least 1300 individual animals per mouse line. Different letters indicate statistically significant differences between lines (Student's $t$-test, $P<0.05)$.

females displayed increased total plasma progesterone concentrations during the cycle, this phenomenon could not be observed in FL1 mice (Fig. 3A; Spitschak et al. 2007). In turn, FL1 females exhibited multiple (up to seven) oocytes per follicle (Alm et al. 2010). Again, this finding is specific for only one FL (FL1) and thus could not be demonstrated for FL2 or unselected control mice.

Additionally, analysis of embryonic survival rates revealed increased gestational losses only in FL1 animals. FL1 females release an average of $\sim 28$ oocytes per cycle, compared with $\sim 12$ oocytes released by Ctrl females (Spitschak et al. 2007). Among this high number of ovulated oocytes, $\sim 18$ develop into living offspring (compare with Table 1). Thus, pregnancy losses are $\sim 1 / 3$ calculated relative to the originally ovulated population. This gestational loss rate is higher compared with that observed in the $\mathrm{Ctrl}(\sim 11 \%$; corresponds to $\sim 11$ offspring per litter - delivered out of $\sim 12$ ovulated oocytes) and FL2 ( $\sim 13 \%$; $~ 20$ offspring - 23 ovulated oocytes). Nevertheless, independent of the fertility strategy selected by the particular mouse line, the improved fecundity lines deliver a substantially higher number of offspring per litter in comparison with Ctrl animals (Table 1). Interestingly, a significantly increased rate of gestational losses (as observed for FL1) has also been described for the Norwegian high-FL (Holt et al. 2004). Moreover, the majority of gestational losses in FL1 animals occur during the first 4 days of pregnancy (Spitschak et al. 2007). It is therefore tempting to speculate that not all of the multiple oocytes that have been ovulated by FL1 females from only one follicle are properly pregnable. As our selection protocol has only focused on first delivery, we are currently forced to speculate whether this 'wasting' phenotype has negative effects on lifetime fecundity performance of our FLs. Furthermore, up to now, we also have no data on further health of the mothers. However, from today's perspective, it is suggested that future health of the females might be impaired due to the strain of huge and heavy litters at first delivery. To clarify these highly crucial issues, further experiments remain to be conducted.

\section{Characterization of the FLs FL1 and FL2 on the male side}

Diallelic breeding experiments indicate a significant contribution of the male side to higher fertility rates. After breeding of male animals from FL1 together with female animals from the Ctrl, significant improved fertility has been observed (Michaelis et al. 2013). Thus, highfertility male contribution seems to largely determine the reproductive outcome. This obviously suggests that not only female ovarian function but also sperm quality of the males and/or heterosis effects of the embryo carry major weight as the limiting factors. This discovered male-only contribution triggered further investigations of FL bucks, which have just started recently.

According to the analysis carried out so far, we could find, on the one hand, a reduced percentage of motile sperm and, on the other hand, increased sperm velocity parameters (such as velocity straight line and velocity average path, among others) compared with control animals (Michaelis et al. 2013). These observations are in contradiction to other findings that typically indicate a higher percentage of motile sperm in fertile characterized animals (Schradin et al. 2012). Furthermore, morphological alterations have been detected in FL1 bucks using a flow cytometric assay with propidium iodide staining (Weitzel et al. 2013). In the testicular parenchyma of these animals, the percentage of haploid cells is decreased with a concomitantly increased percentage of diploid cells (Michaelis et al. 2013). Interestingly, examination of testicular cell-type composition in free-living African striped mice has actually disclosed contrary findings. Corresponding to these data, published by Karin Müller et al., male-dominant and reproductively active African striped mice, which usually live together in colonies with several philopatric, as such reproductively inactive males, exhibit not only
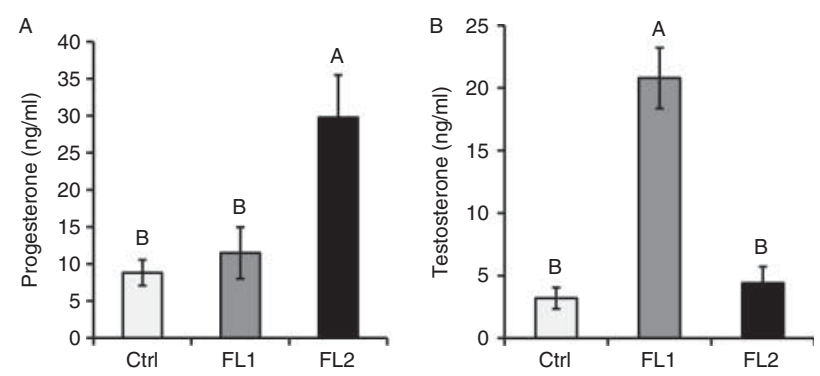

Figure 3 (A) Plasma progesterone concentrations during diestrus in female animals and (B) serum testosterone concentrations in male animals of the fertility lines (FL1 and FL2) and the control line (Ctrl). Data are extracted from the work of Spitschak et al. (2007) and Michaelis et al. (2013) respectively. Different letters indicate statistically significant differences between lines (Student's $t$-test, $P<0.05$ ). 

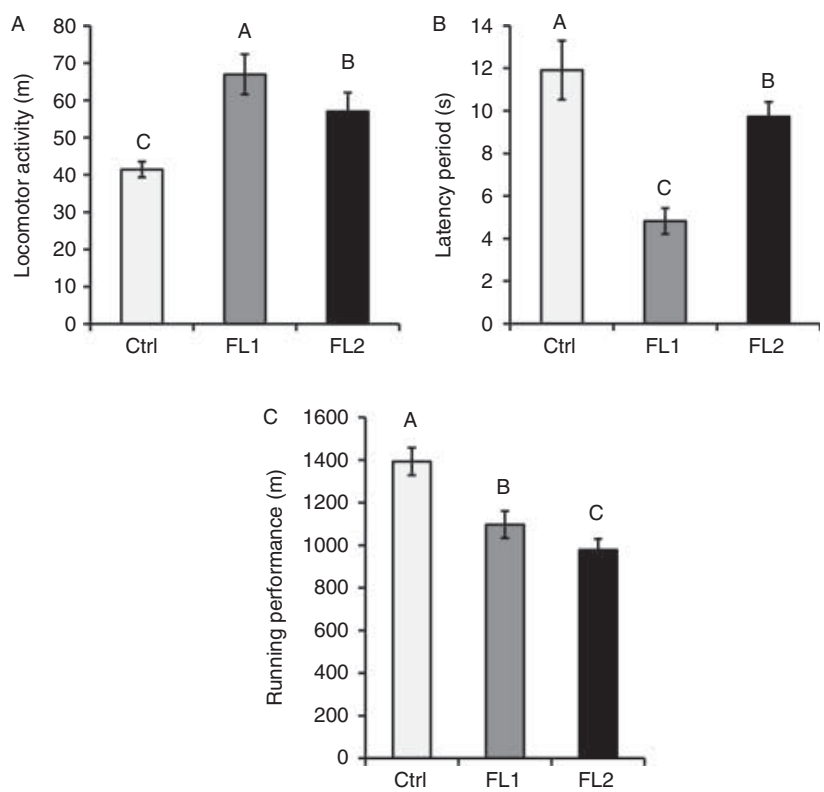

Figure 4 (A) Locomotor activity and (B) latency period in open-field assays for males in both fertility lines (FL1, $n=1013$ and FL2, $n=1033$ ) and in the control line (Ctrl, $n=819)$. Male animals were analyzed on day 70 of life in different generations during the selection period. (A) Distances covered within 3 min were measured in an unknown round surrounding. (B) Initial latency period (in seconds) was analyzed in another open field. (C) Running performance on a treadmill was measured for males of the FLs FL1 $(n=828)$ and FL2 $(n=835)$ as well as the unselected Ctrl $(n=796)$. The endurance fitness was recorded as running distance in meters. Bars represent means \pm s.D. Different letters indicate statistically significant differences between lines (Student's $t$-test, $P<0.05)$.

an increase in haploid cells as well as in motile sperm concentrations, but also an accompanying decline of the diploid cell portion (Raynaud et al. 2012). It might be that different mechanisms are separately and specifically acting to decrease fertility (as in the philopatric mice), to maintain proper fertility (as in the dominant mice), or to induce increased fertility (as in our high-fertility mice).

Additional endocrine blood analyses on FL1 and FL2 bucks have demonstrated dramatically increased total serum testosterone concentrations in FL1 bucks $(20.8 \mathrm{ng} / \mathrm{ml}$; sevenfold higher compared with control animals), whereas FL2 bucks exhibited only slightly raised values $(4.4 \mathrm{ng} / \mathrm{ml})$ (Fig. 3B). In line with this observation, Leydig cell markers have been found to be elevated in the testes of FL1 bucks (Michaelis et al. 2013).

\section{Endocrine and behavioral characteristics}

High testosterone concentrations are often correlated with a higher explorative phenotype (Berenbaum \& Beltz 2011, Eisenegger et al. 2011). Indeed, for our improved FLs, a higher locomotor activity in an open-field experiment could be shown (Renne \& Langhammer 2000). Whereas male control animals covered distances of $\sim 41 \mathrm{~m}$ within $3 \mathrm{~min}$ of analysis, the distance traveled by FL1 animals accounted for $\sim 67 \mathrm{~m}$ in the same time. FL2 bucks, exhibiting only slightly elevated testosterone concentrations compared with control animals, traveled a distance of $\sim 57 \mathrm{~m}$ in this assay (Fig. 4A). In a second open-field study, we measured the latency time in an unknown surrounding. This assay revealed a dramatically reduced latency time for FL1 bucks $(4.8 \mathrm{~s})$ in comparison with FL2 $(9.7 \mathrm{~s})$ as well as control (11.9 s) bucks (Fig. 4B). Thus, male FL1 animals are distinguished by a higher explorative behavior in contrast to FL2 and Ctrl animals.

In another locomotor assay, we tested the endurance fitness of the FL as well as Ctrl males. The running performance, determined on a treadmill (Brenmoehl et al. 2013), was lowered in FL animals compared with control animals (Fig. 4C). Additionally, we noticed that the running performance of all the three lines dropped during the selection process (data not shown). We can currently only speculate about the reasons for this observation. Admittedly, testosterone concentrations have been described to increase during endurance training (Santtila et al. 2009); however, the inverse correlation appears to be un sustainable. Possibly, the decreased running performance reflects an undesired bias due to simultaneously increasing body weight over the selection period (Fig. 2A and data not shown).

Finally, we examined the life expectancy of female and male animals of the FLs in relation to the unselected Ctrl animals. In this assay, females of FL1 did not show any alteration in life expectancy compared with those of the Ctrl, whereas FL2 females exhibited a reduced life span (Fig. 5A). As changes had occurred to a small extent, females of the FLs in general appear to be properly healthy. However, analyses have been carried out on virgin females. Thus, we cannot predict possible negative effects on health and consequently on life expectancy due to the delivery of extremely large and heavy litters.

By contrast, male animals exhibited a different life expectancy with opposing trends in FL1 and FL2.
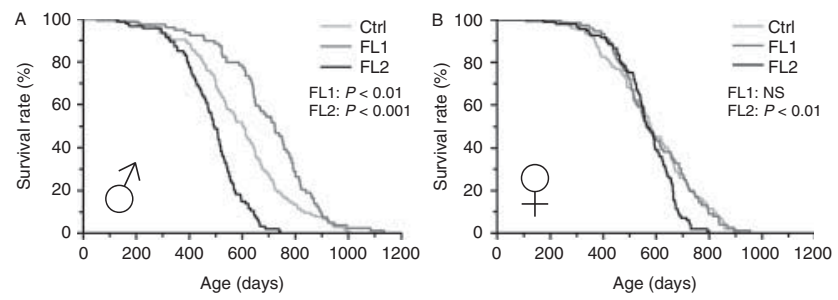

Figure 5 Survival rates of male (A) and female (B) animals of the fertility lines (FL1 and FL2) compared with unselected control animals (Ctrl) shown as Kaplan-Meier plots. The experiment initially started with $n=76$ (FL1), $n=97$ (FL2), and $n=104$ (Ctrl) female mice and $n=79$ (FL1), $n=94$ (FL2), and $n=104$ (Ctrl) male mice. Statistically significant differences for FL1 and FL2 animals relative to Ctrl animals are shown as inset (Student's $t$-test). Animals were housed under standard care conditions. 
Interestingly, FL1 bucks exhibited an increased average life span of about $17 \%$, whereas FL2 males exhibited a life expectancy decrease of around $18 \%$ relative to control animals (Fig. 5B). Obvious explanations for these findings are unfortunately elusive, but it is noteworthy that extremely high testosterone concentrations in FL1 have not elicited any negative effect on life expectancy. By contrast, high testosterone concentrations seem to contribute to an extended life span. Consistently, investigations in humans indicate that higher testosterone concentrations quite correlate with reduced mortality and therefore improved life expectancy (Araujo et al. 2011). Nevertheless, variations in testosterone concentrations in the human study have been subtle alterations within the normal range, whereas FL1 bucks exhibited a sevenfold increased testosterone concentration (Fig. 3B).

\section{Genetic alterations}

Our breeding program over 161 generations implies that multiple genetic alterations might have occurred over this time period. Although we have not addressed this issue experimentally so far, it can be assumed that these genomic modifications are rather polygenetic than monogenetic. This hypothesis is stressed by a recent study comparing seven independent outbred mouse lines selected for high body weight. The breeding protocol of these mice is comparable to our study in spite of differing selection criteria. The analysis indicates that a total of 67 genomic regions have been altered in different mouse lines during selection proceeding (Chan et al. 2012). Additionally, Diethard Tautz et al. could show that the phenotype 'high body weight' based on a smaller subset of genetic variations that had been derived from the pool of 67 genomic regions. Furthermore, it has been striking that each of the seven different mouse lines used another defined subset out of this pool to construct their particular, line-individual genotype (Chan et al. 2012). Thus, the same phenotype (high body weight) is warranted using different genotypes (different genetic alterations). Moreover, another recent genetic screening of an ethnic group referred to as 'Hutterites', distinguished by proscribing contraception and large family sizes, allows the suggestion that 41 genomic regions have been selected for this ethnicity as opposed to an unselected control group (Kosova et al. 2012). Even though both examinations mentioned above are not easy to transfer to our high-fertility mouse models, we nevertheless would suppose that roughly 50-100 genomic regions have been selected and hence modified in our mice.

Presumably, an alteration in every predisposing genomic region is not required to generate, as well as sustain, the improved fertility phenotype. As demonstrated for FL1 and FL2, high fertility is warranted by substantially different molecular strategies in these
Table 2 Overview of different endocrine, physiological, and behavioral phenotypes of the fertility lines FL1 and FL2.

\begin{tabular}{|c|c|c|c|c|}
\hline & \multicolumn{2}{|c|}{ FL1 } & \multicolumn{2}{|c|}{ FL2 } \\
\hline & Male & Female & Male & Female \\
\hline Progesterone & & $\rightarrow$ & & $\uparrow$ \\
\hline Testosterone & $\uparrow \uparrow$ & & $\nearrow$ & \\
\hline $\begin{array}{c}\text { Number of ovulated } \\
\text { oocytes per cycle }\end{array}$ & & $\uparrow \uparrow$ & & $\uparrow$ \\
\hline $\begin{array}{l}\text { Multiple oocytes } \\
\text { per follicle }\end{array}$ & & $\uparrow \uparrow$ & & $\rightarrow$ \\
\hline Embryonic losses & & $\uparrow$ & & $\rightarrow$ \\
\hline Accelerated puberty & & $\uparrow$ & & $\uparrow$ \\
\hline Body weight & $\uparrow$ & $\uparrow$ & $\uparrow$ & $\uparrow \uparrow$ \\
\hline $\begin{array}{l}\text { Locomotor activity } \\
\text { (open field) }\end{array}$ & $\uparrow \uparrow$ & & $\uparrow$ & \\
\hline Running performance & $\searrow$ & & $\searrow$ & \\
\hline Life expectancy & $\uparrow$ & $\rightarrow$ & $\downarrow$ & $\downarrow$ \\
\hline
\end{tabular}

Data are shown relative to the control line animals with the corresponding same gender.

two lines. Consequently, it is reasonable to assume that these lines have been created by a variable combination of different genetic alterations deriving from a pool of predisposing and fertility-relevant genomic regions.

\section{Heterogeneity and biodiversity}

The several physiological, behavioral, and endocrine varieties in the FLs FL1 and FL2 are summarized in Table 2. On the basis of the preceding considerations for the Dummerstorf long-term selection, high-fertility mouse lines, it has become obvious that the advantages of these mouse models are unquestionably their heterogeneity and biodiversity. Thus, the sophisticated genetic background of these outbred mouse lines can shed light on genetic alterations that are associated with highly fertile phenotypes. In contrast to our heterogenetically enhanced FLs, conventional transgenic or knockout mouse models, which merely employ a single-gene variation, constitute a homogenetic basis. Hence, due to their heterogenetic, apparently realistic nature of fertility, our high-fecundity mouse lines offer valuable cues, also for human reproductive medicine.

\section{Declaration of interest}

The authors declare that there is no conflict of interest that could be perceived as prejudicing the impartiality of the research reported.

\section{Funding}

This work was supported by a grant from the German Research Foundation (WE2458/10-1). This article is based on work presented at the 7th Copenhagen Workshop on Endocrine Disrupters, which was supported by the Danish Ministry of the Environment - Environmental Protection Agency. Publication of this special issue was supported by the Society for 
Reproduction and Fertility. None of the authors have any relationship with the Danish Ministry of the Environment Environmental Protection Agency and the Society for Reproduction and Fertility. Joachim Weitzel received travel expenses to attend the COW Workshop from the organizers of the workshop. However, the organizers of the workshop or their sponsors did not have any influence on the experimental setting, data mining, interpretation or presentation of the data presented in this article.

\section{Acknowledgements}

The authors thank the staff of the Mouse Laboratory (LIN), Leibniz Institute for Farm Animal Biology, for conducting the selection experiment, animal care, and sample collection.

\section{References}

Alm H, Kuhlmann S, Langhammer $M$, Tuchscherer A, Torner $\mathbf{H}$ \& Reinsch N 2010 Occurrence of polyovular follicles in mouse lines selected for high fecundity. Journal of Reproduction and Development 56 449-453. (doi:10.1262/jrd.09-224H)

Araujo AB, Dixon JM, Suarez EA, Murad MH, Guey LT \& Wittert GA 2011 Clinical review: Endogenous testosterone and mortality in men: a systematic review and meta-analysis. Journal of Clinical Endocrinology and Metabolism 96 3007-3019. (doi:10.1210/jc.2011-1137)

Beaulieu AD, Aalhus JL, Williams NH \& Patience JF 2010 Impact of piglet birth weight, birth order, and litter size on subsequent growth performance, carcass quality, muscle composition, and eating quality of pork. Journal of Animal Science 88 2767-2778. (doi:10.2527/ jas.2009-2222)

Berenbaum SA \& Beltz AM 2011 Sexual differentiation of human behavior: effects of prenatal and pubertal organizational hormones. Frontiers in Neuroendocrinology 32 183-200. (doi:10.1016/j.yfrne.2011.03.001)

Bowman JC \& Falconer DS 1960 Inbreeding depression and heterosis of litter size in mice. Genetical Research 1 262-274. (doi:10.1017/ S0016672300000240)

Bradford GE 1979 Genetic variation in prenatal survival and litter size. Journal of Animal Science 49 (Suppl 2) 66-74.

Brenmoehl J, Walz C, Renne U, Ponsuksili S, Wolf C, Langhammer M, Schwerin M \& Hoeflich A 2013 Metabolic adaptations in the liver of born long-distance running mice. Medicine and Science in Sports and Exercise 45 841-850. (doi:10.1249/MSS.0b013e31827e0fca)

Chan YF, Jones FC, McConnell E, Bryk J, Bunger L \& Tautz D 2012 Parallel selection mapping using artificially selected mice reveals body weight control loci. Current Biology 22 794-800. (doi:10.1016/j.cub.2012. 03.011)

Dietl G, Langhammer M \& Renne U 2004 Model simulations for genetic random drift in the outbred strain Fzt:Du. Arch Tierzucht 47 595-604.

Eisenegger C, Haushofer J \& Fehr E 2011 The role of testosterone in social interaction. Trends in Cognitive Sciences 15 263-271. (doi:10.1016/ j.tics.2011.04.008)

Holt M, Vangen O \& Farstad W 2004 Components of litter size in mice after 110 generations of selection. Reproduction 127 587-592. (doi:10.1530/ rep.1.00118)

Holt M, Meuwissen T \& Vangen O 2005 The effect of fast created inbreeding on litter size and body weights in mice. Genetics, Selection, Evolution 37 523-537. (doi:10.1186/1297-9686-37-6-523)

Jamsai D \& O'Bryan MK 2011 Mouse models in male fertility research. Asian Journal of Andrology 13 139-151. (doi:10.1038/aja.2010.101)
Johnson RK, Nielsen MK \& Casey DS 1999 Responses in ovulation rate, embryonal survival, and litter traits in swine to 14 generations of selection to increase litter size. Journal of Animal Science 77 541-557.

Kosova G, Scott NM, Niederberger C, Prins GS \& Ober C 2012 Genomewide association study identifies candidate genes for male fertility traits in humans. American Journal of Human Genetics 90 950-961. (doi:10. 1016/j.ajhg.2012.04.016)

Matzuk MM \& Lamb DJ 2008 The biology of infertility: research advances and clinical challenges. Nature Medicine 14 1197-1213. (doi:10.1038/ nm.f.1895)

Michaelis M, Langhammer M, Hoeflich A, Reinsch N, Schoen J \& Weitzel JM 2013 Initial characterization of an outbreed mouse model for male factor (in)fertility. Andrology 1 772-778. (doi:10.1111/j.20472927.2013.00108.x)

Ogorevc J, Dovc P \& Kunej T 2011 Comparative genomics approach to identify candidate genetic loci for male fertility. Reproduction in Domestic Animals 46 229-239. (doi:10.1111/j.1439-0531.2010. 01648.x)

Raynaud J, Muller K \& Schradin C 2012 Experimental increase of testosterone levels in free-ranging juvenile male African striped mice (Rhabdomys pumilio) induces physiological, morphological, and behavioral changes. General and Comparative Endocrinology 178 108-115. (doi:10.1016/j.ygcen.2012.04.028)

Rehfeldt C, Tuchscherer A, Hartung M \& Kuhn G 2008 A second look at the influence of birth weight on carcass and meat quality in pigs. Meat Science 78 170-175. (doi:10.1016/j.meatsci.2007.05.029)

Renne U \& Langhammer M 2000 Effects of long-term selection for exploratory behaviour of mice in an unknown surrounding. Arch Tierzucht 43231.

Santtila M, Kyrolainen H \& Hakkinen K 2009 Serum hormones in soldiers after basic training: effect of added strength or endurance regimens. Aviation, Space, and Environmental Medicine 80 615-620. (doi:10. 3357/ASEM.2479.2009)

Schradin C, Eder S \& Muller K 2012 Differential investment into testes and sperm production in alternative male reproductive tactics of the African striped mouse (Rhabdomys pumilio). Hormones and Behavior 61 686-695. (doi:10.1016/j.yhbeh.2012.03.002)

Schüler L \& Bünger L 1982 Die reproduktive Lebensleistung auf Fruchtbarkeit selektierter Labormauslinien. Arch Tierzucht 25 275-281.

Silver LM 1995 In Mouse Genetics - Concepts and Applications, ch 4.1. Oxford: Oxford University Press.

Spitschak M, Langhammer M, Schneider F, Renne U \& Vanselow J 2007 Two high-fertility mouse lines show differences in component fertility traits after long-term selection. Reproduction, Fertility, and Development 19 815-821. (doi:10.1071/RD07009)

Su G, Lund MS \& Sorensen D 2007 Selection for litter size at day five to improve litter size at weaning and piglet survival rate. Journal of Animal Science 85 1385-1392. (doi:10.2527/jas.2006-631)

Wei J, Ramanathan P, Thomson PC, Martin IC, Moran C \& Williamson P 2013 An integrative genomic analysis of the superior fecundity phenotype in QSi5 mice. Molecular Biotechnology 53 217-226. (doi:10.1007/s12033-012-9530-y)

Weitzel JM, Vernunft A, Kruger B, Plinski C \& Viergutz T 2014 LOX-1 regulates estrogenesis via intracellular calcium release from bovine granulosa cells. Cytometry. Part A 85 88-93. (doi:10.1002/cyto.a.22353)

Ziadi C, Moce ML, Laborda P, Blasco A \& Santacreu MA 2013 Genetic selection for ovulation rate and litter size in rabbits: estimation of genetic parameters and direct and correlated responses. Journal of Animal Science 91 3113-3120. (doi:10.2527/jas.2012-6043)

Received 5 September 2013

First decision 7 October 2013

Revised manuscript received 7 November 2013

Accepted 18 November 2013 